\title{
A novel index to detect green-tide using UAV-based RGB imagery
}

\author{
Xiaopeng Jiang a,b,c,d, Meng Gao ${ }^{\text {a,b,d, Zhiqiang Gao }}{ }^{\text {a,b,d, * }}$ \\ ${ }^{a}$ CAS Key Laboratory of Coastal Environmental Processes and Ecological Remediation, Yantai Institute of Coastal Zone Research, Chinese Academy of Sciences, Yantai \\ Shandong, 264003, PR China \\ ${ }^{\mathrm{b}}$ Shandong Key Laboratory of Coastal Environmental Processes, Yantai Institute of Coastal Zone Research, Chinese Academy of Sciences, Yantai, 264003, PR China \\ ${ }^{\mathrm{c}}$ University of Chinese Academy of Sciences, Beijing, 100049, PR China \\ ${ }^{\mathrm{d}}$ Center for Ocean Mega-Science, Chinese Academy of Sciences, Qingdao, Shandong, 266400, PR China
}

\section{A R T I C L E I N F O}

\section{Keywords:}

Unmanned aerial vehicle (UAV)

RGB-FAI

Remote sensing

Green tide

Drift velocity estimation

\begin{abstract}
A B S T R A C T
Unmanned aerial vehicles (UAV) equipped with high-resolution camera have been increasingly applied in environment monitoring as an important complement to traditional satellite remote sensing. An accurate extraction of marine green-tide regions still faces many technological challenges, such as the absence of centimeter-level orthophoto maps and a dedicated green-tide index based on red-green-blue (RGB) bands. In this study, a new green-tide index, namely, the red-green-blue floating algae index (RGB-FAI) using RGB images captured by ship-borne UAV, is developed for green-tide detection in the Yellow Sea, China. Specifically, RGBFAI is defined to measure the green-reflectance height by using the red and blue bands as the baselines. Our results show that the RGB-FAI performs well in the detection of green-tide and the accuracy is satisfactory (kappa $=0.95$ ). It is worthy to note that RGB-FAI has the highest extraction accuracy among these competing indices for green-tide in the declining phase under a hazy atmospheric condition. In addition, by combining the bi-temporal UAV images with RGB-FAI, the drift velocity of green-tide has also been estimated as $0.26 \mathrm{~m} / \mathrm{s}$ in a $17.1^{\circ}$ east by north during aerial photography. In conclusion, the proposed RGB-FAI is effective for green-tide detection and has more potential usage in marine environment monitoring.
\end{abstract}

\section{Introduction}

Massive green-tides in the Yellow Sea, a form of harmful macroalgal blooms of Ulva.prolifera (Liu et al., 2013, 2016b; Zhao et al., 2013), have appeared continuously for 14 years since 2007 (Zhou et al., 2015) causing serious environmental problems and economic losses (Liu et al., 2009; Smetacek and Zingone, 2013; Zhao et al., 2018). Recurring green-tides have induced a series of negative impacts on the marine ecosystem (Liu et al., 2010) and been considered as an ecological disaster by the Chinese government (Yu et al., 2018). As such, many measures have been taken to monitor and prevent green-tide disasters (Yan et al., 2018). The area and biomass of green-tides are two critical bloom parameters for disaster prevention and control (Wang et al., 2018). Generally, bloom information such as area and migration trajectory can be retrieved by using multi/hyper-spectral satellite images combined with vegetation indices (Son et al., 2012; Bao et al., 2015).

Vegetation indices are combinations of reflectance at different bands used to highlight vegetation information. The classic vegetation indices such as the normalized difference vegetation index (NDVI) (Rouse et al., 1974) and enhanced vegetation index (EVI) (Huete et al., 1999) have been proposed and utilized to map vegetative cover (Luo et al., 2005). Analogously, some scholars also proposed ocean color indices aimed for detecting green-tidal blooms. Among these, the seminal work is the floating algae index (FAI) proposed in (Hu, 2009), and has been widely used in these subsequent studies (Cui et al., 2012; Oyama et al., 2015; Hu et al., 2017). Moreover, to solve the problem the HJ-1 application problems of FAI due to a lack of shortwave infrared bands, the virtual-baseline floating macro-algae height (VB-FAH) (Xing and Hu, 2016) was developed. VB-FAH could be applied to higher-resolution (30 m) satellite images.

However, NDVI, EVI, FAI, VB-FAH, and other indices are all satellitebased multi/hyper-spectral images but not suitable for red-green-blue (RGB) aerial images. In recent years, unmanned aerial vehicles (UAV) have become a beneficial supplement to ground surveys and satellite remote sensing. The advantages of UAV could be concluded as low cost, flexibility, and high ground resolution (Hassan et al., 2019). Therefore,

\footnotetext{
* Corresponding author. CAS Key Laboratory of Coastal Environmental Processes and Ecological Remediation, Yantai Institute of Coastal Zone Research, Chinese Academy of Sciences, Yantai Shandong, 264003, PR China.

E-mail address: zqgao@yic.ac.cn (Z. Gao).
} 

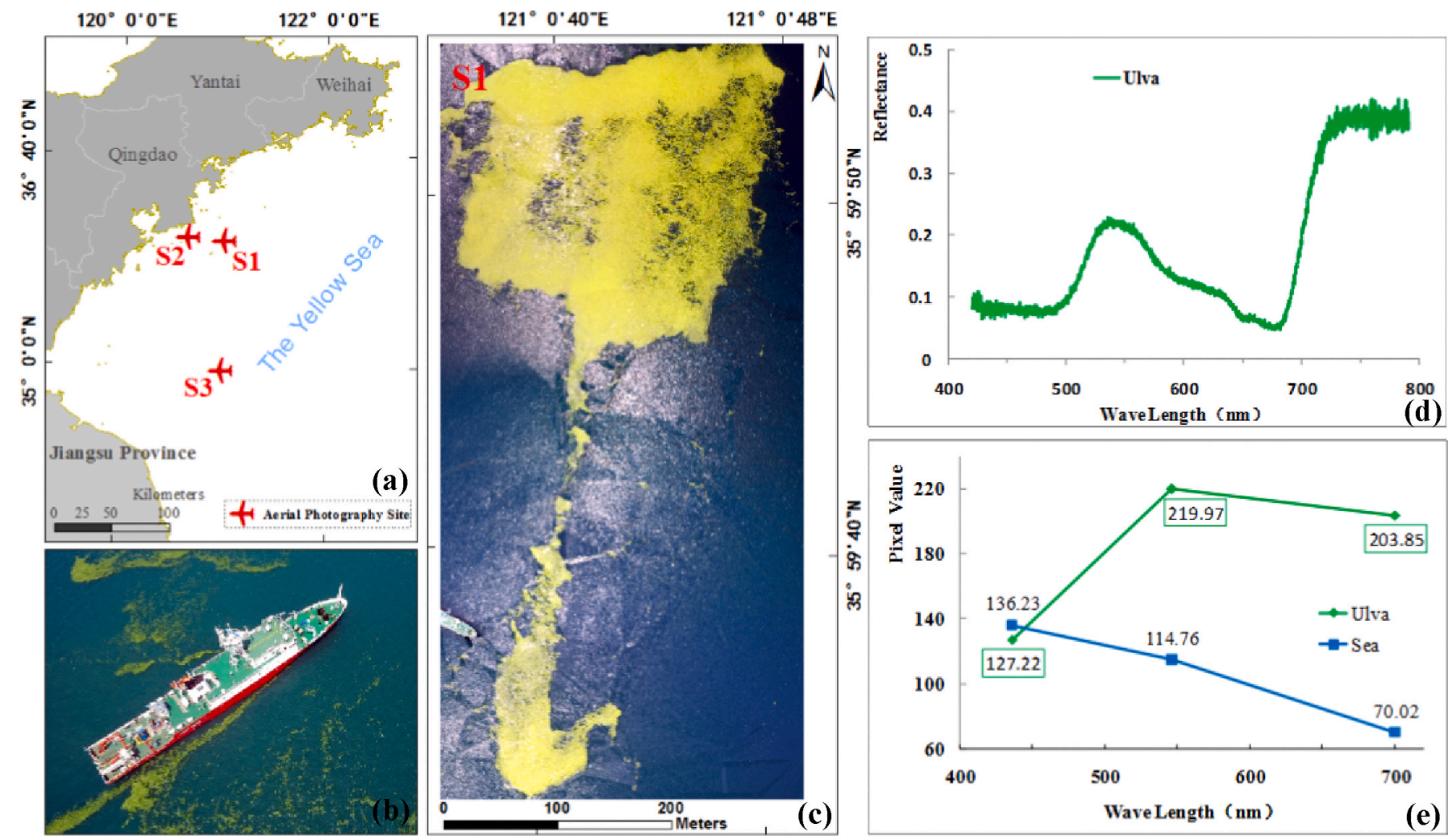

Fig. 1. (a) Study area and three aerial photography sites: S1, S2, and S3, indicated by a red symbol; (b) the research vessel and floating green algae on the sea, shot by unmanned aerial vehicle (UAV); (c) Digital orthophoto map of green-tide algae (Ulva.prolifera) at site S1 (containing sun glint); (d) reflectance spectra of U.prolifera, measured by USB 4000 spectrometer; and (e) the pixel mean values of U.prolifera and seawater in red-green-blue bands, derived from Fig. 1c. (For interpretation of the references to color in this figure legend, the reader is referred to the Web version of this article.)

the UAV applications were wide ranging from environment protection (Fallati et al., 2019), disaster relief (Erdelj et al., 2017) to agricultural production(Kim et al., 2019). At present, multi-spectral sensors suitable for UAV are still expensive and heavy. Most mainstream UAVs are equipped with RGB digital cameras (Zhang et al., 2019). RGB vegetation indices are few and originally designed for land vegetation (Wang et al., 2015a). The pathways of the green-tide in the Yellow Sea are so far away from land (Bao et al., 2015) that the land-based UAV aerial photography is nearly impossible. In addition, to our best knowledge there is no dedicated green-tide index suitable for RGB bands. Therefore, accurate and timely detection of green-tide via UAV was almost omitted in previous studies.

In this study, a consumer UAV equipped with RGB digital cameras was applied to obtain aerial images of green-tide with the marine survey ship as the landing pad. A new green-tide index, the red-green-blue floating algae index (RGB-FAI), was proposed based on the baseline subtraction method. Then, RGB-FAI was compared with several published RGB vegetation indices to show its extraction capability. Finally, RGB-FAI functions including estimating the drift velocity and monitoring scattered small patches of the green-tide were presented and discussed in the final two sections.

\section{Material and methods}

\subsection{Acquisition and processing of UAV-based RGB images}

The study area is located in the South Yellow Sea, China (Fig. 1a), which is mainly affected by the East Asian monsoon, with south/ southeast wind prevailing in summer and north wind prevailing in autumn and winter. The western part of the South Yellow Sea has a regular semidiurnal tidal cycle e (Chen et al., 2018). The inter-annual variation of chlorophyll concentration is significant in this sea, and the spatial distribution shows a decreasing trend from the near-shore to the middle of the Yellow Sea. The maximum value of chlorophyll concentration appears in April and the minimum value appears in June or July (Tian et al., 2020).

The UAV applied in this study is the DJI Inspire 1 UAV (SZ DJI Technology Co., Ltd., Shenzhen, China) taking the deck of research

Table 1

Details of digital orthophoto maps used in this study. BJT: Beijing time (UTC+8).

\begin{tabular}{|c|c|c|c|c|c|c|c|c|}
\hline Site & $\begin{array}{l}\text { Operation Start } \\
\text { Time (BJT) }\end{array}$ & $\begin{array}{l}\text { Center } \\
\text { Location }\end{array}$ & $\begin{array}{l}\text { UAV Flight } \\
\text { Height (m) }\end{array}$ & $\begin{array}{l}\text { Spatial } \\
\text { resolution (m) }\end{array}$ & $\begin{array}{l}\text { Life phase of } \\
\text { green algae }\end{array}$ & $\begin{array}{l}\text { Weather } \\
\text { conditions }\end{array}$ & Area $\left(\mathrm{m}^{2}\right)$ & Roles \\
\hline \multirow[t]{2}{*}{ S1 } & 19/06/19 13:04 & $\begin{array}{l}36^{\circ} \mathrm{N}, 121^{\circ} \\
\mathrm{E}\end{array}$ & 320 & 0.12 & Growth phase & $\begin{array}{l}\text { Clear } \\
\text { atmosphere }\end{array}$ & $317 * 662$ & $\begin{array}{l}\text { For Section } 2.2 \text { and } 3.1 \text { : Index design } \\
\text { and evaluation of its accuracy }\end{array}$ \\
\hline & & & & & & & $700 * 2400$ & $\begin{array}{l}\text { For Section } 4.2 \text { : Calculation of green- } \\
\text { tide drift velocity }\end{array}$ \\
\hline S2 & $19 / 07 / 23$ 08:03 & $\begin{array}{l}36^{\circ} \mathrm{N} \\
120.7^{\circ} \mathrm{E}\end{array}$ & 400 & 0.15 & Growth phase & $\begin{array}{l}\text { Clear } \\
\text { atmosphere }\end{array}$ & $460 * 835$ & For Section 3.2: Verification of RGB-FAI \\
\hline S3 & 19/07/25 07:09 & $\begin{array}{l}35^{\circ} \mathrm{N}, 121^{\circ} \\
\mathrm{E}\end{array}$ & 265 & 0.09 & Decline phase & $\begin{array}{l}\text { Hazy } \\
\text { atmosphere }\end{array}$ & $682 * 348$ & $\begin{array}{l}\text { For Section 4.1: Extraction of the } \\
\text { declining green-algae }\end{array}$ \\
\hline
\end{tabular}




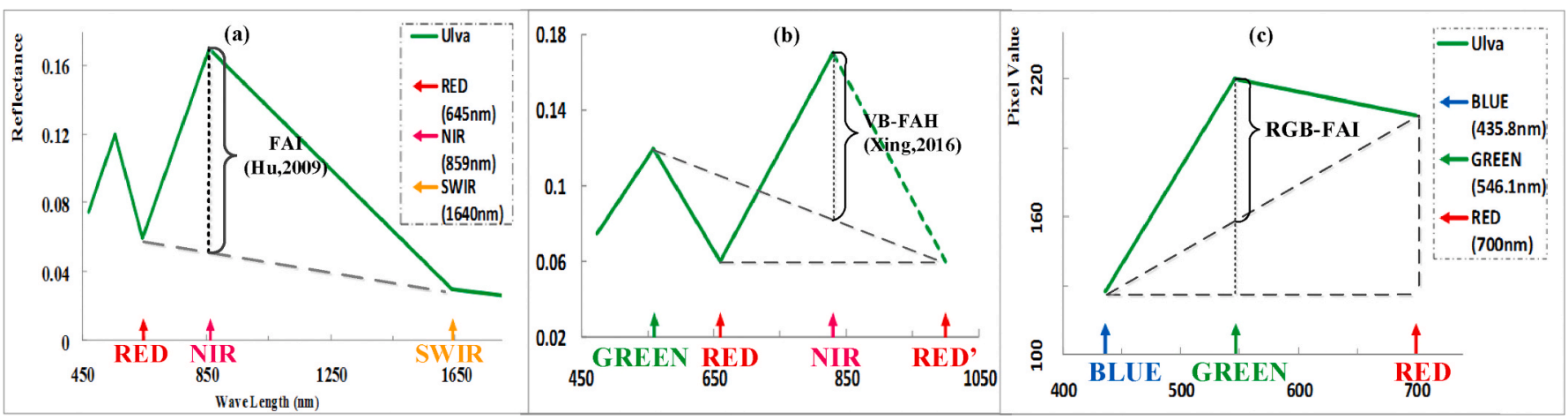

Fig. 2. Illustration of (a) floating algae index (FAI) (Hu, 2009), (b) virtual baseline floating algae height (VB-FAH) (Xing and Hu, 2016), and (c) the red-green-blue floating algae index(RGB-FAI). (For interpretation of the references to color in this figure legend, the reader is referred to the Web version of this article.)

vessel (Fig. 1b, KE XUE SAN HAO, Institute of Oceanology, Chinese Academy of Sciences) as the landing pad It was equipped with a 12.4megapixel RGB camera, adopting a global navigation and positioning system to incorporate geographic coordinates and flight height into aerial photos. During two voyage surveys from June to July 2019, aerial photographs of the green-tide from three sites (Fig. 1a) were selected and applied to this study. As very-low altitude remote sensing technology, the UAV image is minimally affected by the Earth's rotation and atmospheric disturbances (Xing et al., 2010). Pix4Dmapper (Pix4D SA, Lausanne, Switzerland) was used to generate the digital orthophoto map of the green algae patches. The details of the othophoto images from three sites in this study are shown in Table 1 . Their weather conditions and life phase of green algae are different, and the three images have different usage.

\subsection{Construction of RGB-FAI}

The dominant species causing the green-tide in this study is U.prolifera, which is a kind of large floating algae on the sea surface. Thus, as shown in Fig. 1d and derived using a USB4000 micro-spectrometer (Ocean Optics Inc, USA), the reflectance spectrum of U.prolifera is generally similar to that of land vegetation, with high reflection in green and near-infrared bands and absorption in blue and red bands. The UAVbased RGB images of the study site S1 (Fig. 1c) mainly contain green algae and seawater. Twenty sample areas of the two types were selected, respectively, to calculate the pixel mean value of green algae and seawater in the red, green, and blue bands. The statistical results (Fig. 1e) show that the pixel values of U.prolifera satisfy $R_{\text {green }}>R_{\text {red }}>$ $\mathrm{R}_{\text {blue }}$, that is, U.prolifera had the highest reflectance in the green band, followed by the red band, and the blue band, which is consistent with the spectral curve in Fig. 1d. The spectral characteristic of seawater is $\mathrm{R}_{\text {blue }}>\mathrm{R}_{\text {green }}>\mathrm{R}_{\text {red }}$. In addition to a small difference in the blue band, the reflectance of seawater in the green and red bands was far lower than that of U.prolifera.

To detect the floating algae accurately, Hu (2009) introduced the FAI using the baseline-subtraction approach:

$\mathrm{FAI}=\mathrm{R}_{\mathrm{NIR}}-\mathrm{R}_{\text {red }}-\left(\mathrm{R}_{\mathrm{SWIR}}-\mathrm{R}_{\mathrm{red}}\right) *\left(\lambda_{\mathrm{NIR}}-\lambda_{\text {red }}\right) /\left(\lambda_{\text {SWIR }}-\lambda_{\text {red }}\right)$,

where $R$ is the reflectance and $\lambda$ is the wavelength; the subscripts $N I R$, red, and SWIR represent the near-infrared (NIR), red, and shortwave infrared (SWIR) bands, respectively. The FAI uses the red band and SWIR bands as the baseline to measure the height of the NIR reflectance (Fig. 2a). Similarly, based on the baseline subtraction method, Xing et al. (Xing and Hu, 2016) developed the VB-FAH index (Fig. 2c) for mapping floating algae using the NIR, red, and green bands. FAI and VB-FAH are not sensitive to changes in the solar/viewing geometry and atmospheric conditions as well as other perturbations (e.g. thin clouds), thus making it more efficient in detecting floating algae. Both indices have been widely utilized to study green-tide due to their effectiveness and simplicity. However, neither index can be applied to UAV-based RGB images due to lacking NIR and SWIR bands. Therefore, an alternative index should be introduced to take advantage of the designs of FAI and VB-FAH.

Here, considering both the spectrum curve of U.prolifera (Fig. 1d and e) and principle of the baseline subtraction method (e.g. FAI or VBFAH), a novel index for detecting green-tide algae using RGB images is developed and named the Red-Green-Blue Floating Algae Index (RGBFAI). The RGB-FAI uses two bands in the blue and red to form a baseline, which is then subtracted from the green reflectance. Fig. 2 illustrates the conceptions of FAI, VB-FAH, and RGB-FAI, respectively. The RGB-FAI is defined as:

$\mathrm{RGB}-\mathrm{FAI}=\mathrm{R}_{\text {green }}-\mathrm{R}_{\text {blue }}-\left(\mathrm{R}_{\text {red }}-\mathrm{R}_{\text {blue }}\right) *\left(\lambda_{\text {green }}-\lambda_{\text {blue }}\right) /\left(\lambda_{\text {red }}-\lambda_{\text {blue }}\right)$,

where $R$ is the reflectance and $\lambda$ is the wavelength. The subscripts red, green, and blue represent the red, green, and blue bands, respectively. Defined by the International Commission on Illumination (CIE) 1931 standard colorimetric system, $\lambda_{\text {red }}=700 \mathrm{~nm}, \lambda_{\text {green }}=546.1 \mathrm{~nm}, \lambda_{\text {blue }}=$ $435.8 \mathrm{~nm}$ (Fairman et al., 1997). As shown in Fig. 1d and e, U.prolifera displayed specific spectral characteristics namely, higher green reflectance than seawater and higher green reflectance than in the red and blue bands. These features provide the basis of the RGB-FAI design; while the green band is used to measure the height, the blue and red bands act as the baseline. This is similar to the band-subtraction design of FAI and VB-FAH.

\subsection{Extracting green-tide from $U A V$-based $R G B$ images}

The new RGB-FAI index was evaluated by testing against another five RGB vegetation indices. There are three main steps to extract floating green algae from remote sensing images: first, it is necessary to calculate the vegetation index of the images; second, a threshold in the histogram of vegetation index images must be set to distinguish green algae from non-green algae; and finally, it is necessary to calculate the accuracy of the classification results.

\section{1) The RGB vegetation indices}

Most of the current vegetation indices are based on the visible-near infrared bands derived from multi/hyper-spectral sensors. RGB band vegetation indices are few and include excess green (EXG, Eq. (2)) (Bellvert et al., 2014), the normalized green-blue difference index (NGBDI, Eq. (3)) (Hunt et al., 2005), normalized green-red difference index (NGRDI, Eq. (4)) (Meyer and Neto, 2008), red-green-blue vegetation index (RGBVI, Eq. (5)) (Bendig et al., 2015), and visible-band difference vegetation index (VDVI, Eq. (6)) (Wang et al., 2015a). In 




(a)

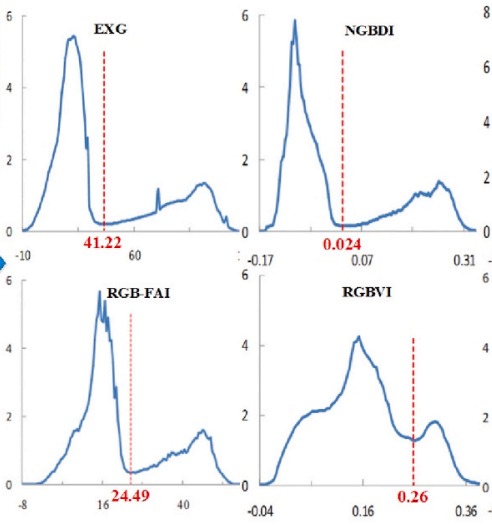

(b)
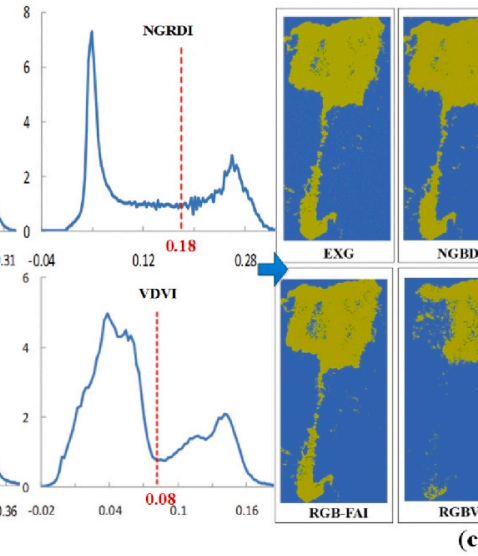

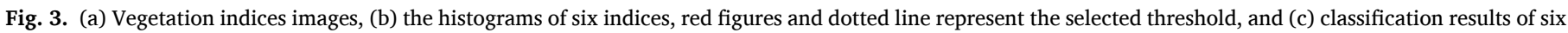
vegetation indices. (For interpretation of the references to color in this figure legend, the reader is referred to the Web version of this article.)

this study, these indices are compared to validate the newly proposed index for green-tide extraction.

$\mathrm{EXG}=2 \mathrm{R}_{\text {green }}-\mathrm{R}_{\text {red }}-\mathrm{R}_{\text {blue }}$

$\mathrm{NGBDI}=\mathrm{R}_{\text {green }}-\mathrm{R}_{\text {blue }} /\left(\mathrm{R}_{\text {green }}+\mathrm{R}_{\text {blue }}\right)$

$\mathrm{NGRDI}=\mathrm{R}_{\text {green }}-\mathrm{R}_{\mathrm{red}} /\left(\mathrm{R}_{\text {green }}+\mathrm{R}_{\mathrm{red}}\right)$

$\mathrm{RGBVI}=\mathrm{R}_{\text {green }}^{2}-\mathrm{R}_{\text {red }} * \mathrm{R}_{\text {blue }} /\left(\mathrm{R}_{\text {green }}^{2}+\mathrm{R}_{\text {red }} * \mathrm{R}_{\text {blue }}\right)$

VDVI $=2 R_{\text {green }}-R_{\text {red }}-R_{\text {blue }} /\left(2 R_{\text {green }}+R_{\text {red }}+R_{\text {blue }}\right)$,

where $R_{\text {red }}, R_{\text {green }}$, and $R_{\text {blue }}$ represent the reflectance or pixel values of the red, green, and blue bands, their wavelengths are also $700 \mathrm{~nm}$, $546.1 \mathrm{~nm}$, and $435.8 \mathrm{~nm}$, respectively.

\section{2) Determining a threshold for classification}

The selection of a threshold is a key factor affecting result accuracy. In this study, as the vegetation index images have bimodal characteristics, the bimodal histogram method was applied. The value corresponding to the trough in the histogram was determined as the threshold.

\section{3) Accuracy assessment}

The kappa coefficient is a robust statistical method used to assess the agreement between the classification result and reference images (Pontius and Millones, 2011). In this study, in order to reduce the error caused by randomness, 1000 random verification points were generated to almost completely cover the image (see Supplemental Fig. S1). Then all points were labeled under their actual type (green algae or seawater) by referring to the clear original images. Finally, by comparing the classification results of six indices with reference points using ENVI software, the confusion matrix and Kappa coefficient were obtained to evaluate the classification results.

The kappa coefficient is divided into six groups corresponding to different rating criteria: $1-0$ (poor agreement), $0.01-0.20$ (slight agreement), $0.21-0.40$ (fair agreement), $0.41-0.60$ (moderate agreement), $0.61-0.80$ (substantial agreement), and $0.81-1.00$ (almost perfect agreement); kappa $=1$ implies perfect agreement (Rosenfield and Fitzpatrick-Lins, 1986).
Table 2

Accuracy assessment of the six classified images.

\begin{tabular}{llll}
\hline \multirow{2}{*}{ Vegetation indices } & \multicolumn{2}{l}{ Accuracy/\% } & \multirow{2}{*}{ Kappa coefficient } \\
\cline { 2 - 3 } & Green algae & Seawater & \\
\hline EXG & 98.35 & 99.28 & 0.976 \\
NGBDI & 99.34 & 98.28 & 0.967 \\
NGRDI & 99.34 & 76.15 & 0.655 \\
RGB-FAI & 94.39 & 99.43 & 0.950 \\
RGBVI & 57.1 & 99.86 & 0.647 \\
VDVI & 92.74 & 98.13 & 0.916 \\
\hline
\end{tabular}

\section{Result and analysis}

\subsection{Extraction results and accuracy evaluation}

According to the formula of the RGB vegetation indices (Eq. (1)-(6)), six vegetation indices of UAV-based RGB images (Fig. 1c) were calculated to obtain the vegetation indices images (Fig. 3a) by the ENVI software. Among them, the value range of NGBDI, NGRDI, RGBVI, and VDVI is $[-1,1]$, and the value range of EXG and RGB-FAI is [-255, 255]. Subsequently, the histograms of six vegetation index images were generated (Fig. 3b).

In Fig. 3b, the abscissa is the value of the vegetation indices, and the ordinate is the pixel count. The histograms of six vegetation indices all have bimodal characteristics. Therefore, the bimodal histogram threshold method was used to determine the thresholds, as shown by the red figures and dotted lines in Fig. 3b. These selected thresholds were used to extract the floating green algae, then, the classification results of six vegetation indices were generated (Fig. 3c).

The accuracy assessment results from the confusion matrix of six classified images were shown in Table 2.

According to Table 2 and the rating criteria of the kappa coefficient, the classification result of the RGB-FAI (kappa $=0.95$ ) indicates very close to perfect agreement. Moreover, RGB-FAI, EXG, and NGBDI have the best levels of green algae extraction (kappa $\geq 0.95$ ). Although RGBFAI is not the most accurate, the gap between their kappa coefficients is extremely small (a difference of only 0.026); the precision of VDVI ranks secondly. The extraction accuracy of neither NGRDI nor RGBVI is poor, with the lowest kappa coefficients. NGRDI can extract most of the green algae but the odds of seawater being mistaken for green algae are great.

\subsection{Verification of RGB-FAI}

Section 3.1 shows that RGB-FAI has high accuracy in the extraction of floating green algae. To verify the applicability and reliability of RGB- 

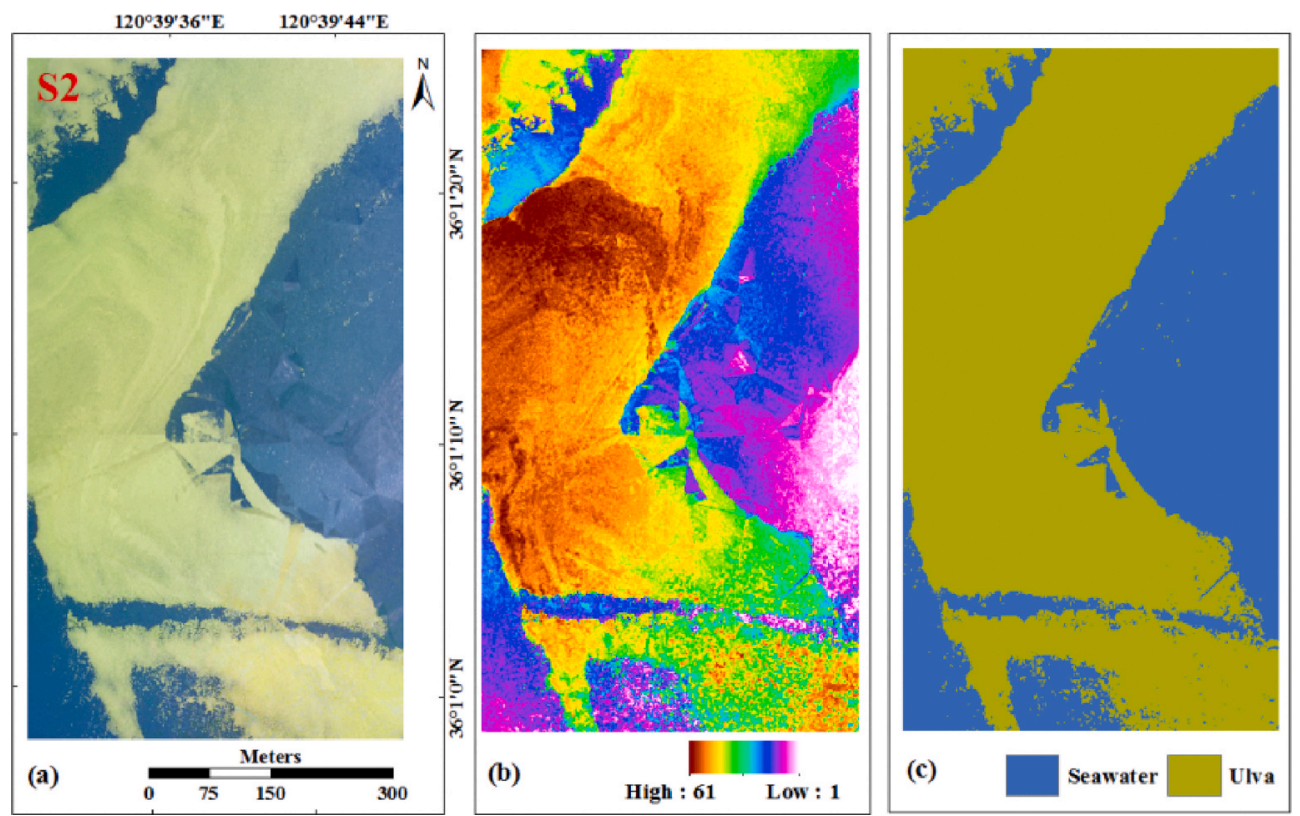

Fig. 4. (a) UAV-based digital orthophoto map of green-tide at site S2 under a clear atmosphere; (b) vegetation index image of RGB-FAI; (c) classified image of RGBFAI. (For interpretation of the references to color in this figure legend, the reader is referred to the Web version of this article.)

Table 3

Confusion matrix of classified image using RGB-FAI.

\begin{tabular}{lllll}
\hline & Seawater & Green algae & Row total & User accuracy/\% \\
\hline Seawater & 361 & 16 & 377 & 95.76 \\
Green algae & 7 & 616 & 623 & 98.88 \\
Column total & 368 & 632 & 1000 & \\
$\begin{array}{l}\text { Producer accuracy/ } \\
\text { \% }\end{array}$ & 98.10 & 97.47 & & \\
\hline
\end{tabular}

FAI, another group of UAV-based RGB images of floating green algae from site S2 (see Fig. 1a) were selected to generate the digital orthophoto map of the green-tide (Fig. 4a). The details for this group of images are shown in the third row of Table 1(Site: S2). Based on the same process, RGB-FAI was applied to extract the green algae. The vegetation index image (Fig. 4b) and classified image (Fig. 4c) of RGB-FAI were generated successively. The accuracy report from the confusion matrix of the classified image was tabulated in Table 3. The producer and user accuracies were obtained and are also shown in this table.
According to Table 3, an overall accuracy and kappa coefficient were calculated for the RGB-FAI, with an overall accuracy of $97.7 \%$ and kappa coefficient of 0.951 . These accuracy results imply that the classification results almost perfectly concur with reality. Combining the results of Section 3.1 and this section, the RGB-FAI can meet the requirements for high-precision extraction of green-tide from UAV-based RGB images (kappa $\approx 0.950$ ).

\section{Discussion}

\subsection{Accurate extraction of scattered small patches of green-tide in a hazy atmosphere}

From July to August, the green-tide in the Yellow Sea entered into the decline phase. Compared with the previous growth phase, the chlorophyll content of the U.prolifera decreased, the color of the algae changed from green to light green or yellow-green, the cell spacing increased, and some cells became granular (Liu et al., 2016a). Changes in pigment and cell structure directly affect the spectral characteristics
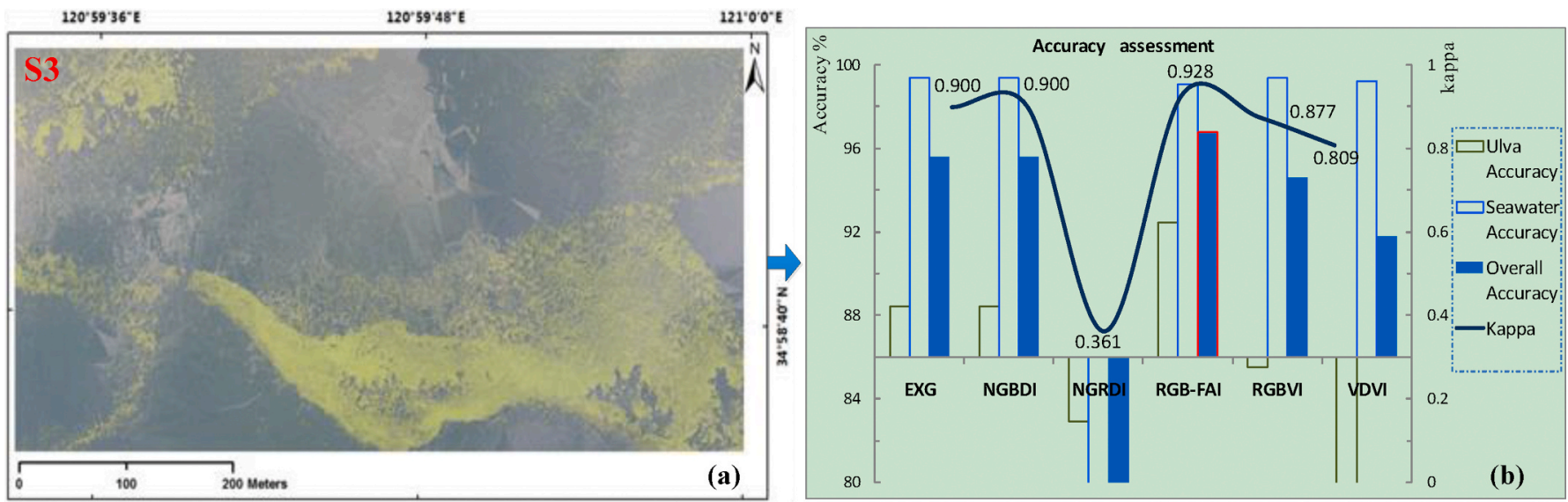

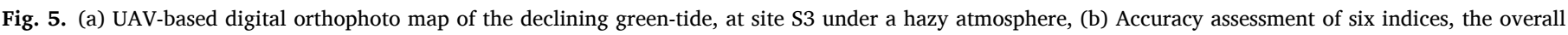

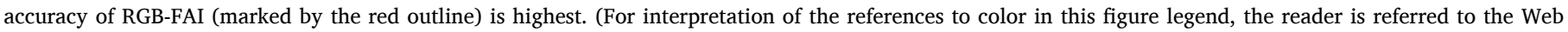
version of this article.) 
of U.prolifera. The large-area patches that formed in the previous growth phase also separated into several scattered smaller patches due to aging and sedimentation of the U.prolifera. These factors increase the difficulty of extracting the $U$.prolifera by remote sensing.

Large bands or patches of floating green algae are easily detected by satellite remote sensing. However, during the early and decline phases of green-tide, scattered and small areas of green algae patches exist in large numbers (Wang et al., 2015b). Even in its development phase, there were many small-area patches around the large patch (see Supplemental Fig. S2). Whereas the MODIS (250 m resolution) cannot adequately monitor the scattered green algae patches. When HJ-1 (30 m resolution) is used as the reference, the extraction area of the green-tide using MODIS images may be exaggerated by $21 \%$ (Zhong et al., 2013). The mixed pixel effect, caused by insufficient image spatial resolution, will cause large deviations in the extraction results of different satellites (Zheng et al., 2017). UAV remote sensing can acquire centimeter-level spatial resolution images. When combined with the RGB-FAI, scattered and small patches can be accurately detected, making up for the shortages of satellite remote sensing.

On the morning of July 25, 2019 (Beijing time), under hazy weather, aerial photographs of the declining U.prolifera at Site S3 (see Fig. 1a and Table 1), with scattered distribution and yellow-green color, were taken by UAV. Using the same process, a digital orthophoto map with a spatial resolution of $9 \mathrm{~cm}$ was generated (Fig. 5a), then, six vegetation indices (Eq. (1)-(6)) were used to extract the U.prolifera from the digital orthophoto map (see Supplemental Fig. S3). Finally, the accuracy of the six extraction results was evaluated. The accuracy assessment results containing overall accuracy and kappa coefficient are presented in Fig. 5b.

As shown in Fig. 5b, RGB-FAI has the highest accuracy for the extraction of green algae (kappa $=0.928$, which is better than the other indexes). The accuracy of EXG and NGBDI is still relatively high (kappa $=0.900$ ), exceeding the other three vegetation indices. This shows that under the low-visibility atmosphere, RGB-FAI has a unique advantage in extracting the declining green-tide.

In addition, under clear atmospheric conditions, the green-tide extraction accuracy of RGB-FAI (kappa $=0.95$ ) is only slightly lower than the highest EXG, with the kappa gap just 0.026; While under the low-visibility conditions, RGB-FAI had the highest accuracy compared to other tested indices. It proved that RGB-FAI, similar to the FAI that also adopting the baseline correction idea, can improve performance in avoiding of perturbations from aerosols.

\subsection{Calculation of green-tide drift velocity based on RGB-FAI}

Several studies have shown that the green-tide in the Yellow Sea originate in the Subei Shoal (southern Yellow Sea, China) (Liu et al., 2009, 2012; Zhang et al., 2018), then drift northward to the south coast of the Shandong peninsula (Liu et al., 2013; Wang et al., 2015b). As a trans-regional disaster, the drift velocity of green-tide has always been the focus of disaster prevention and mitigation (Cui et al., 2012). Satellite remote sensing is only effective when there is little or cloud cover in the sky and the shape of green-tidal patches change greatly every day, making it difficult to successfully track and identify the same patch for satellite images with a revisit period greater than one day (Chen et al., 2018). Thus, does not meet the real-time monitoring needs of green-tide disasters. At this stage, the accuracy of monitoring green-tidal distribution and dynamic changes using satellite images is low (Wang et al., 2018).

The aerial orthophotos of the green-tide in the Yellow Sea can be acquired conveniently using ship-borne UAV. This option avoids clouds and is flexible in application. Moreover, it can continuously track the floating green algae patches, thereby obtaining aerial orthophotos of the same green algae patches at different times. The drift velocity can be calculated using the RGB-FAI to extract the displacement of green algae patches with the capture time recorded in aerial photographs.

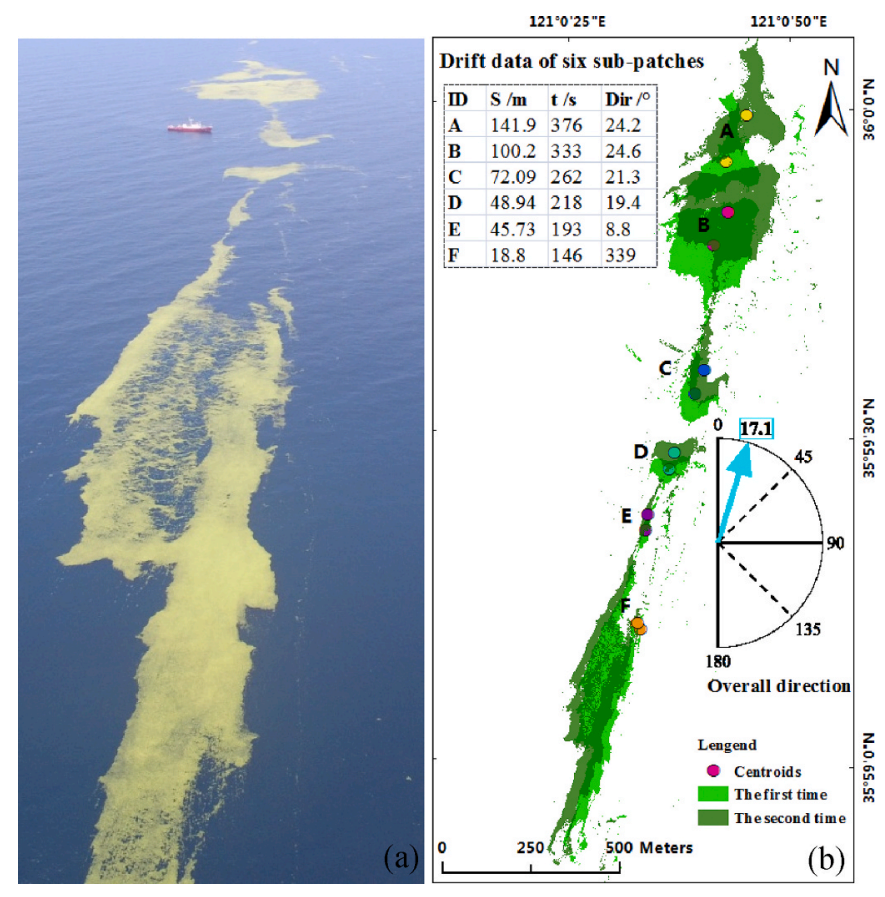

Fig. 6. (a) Side view aerial photography and (b) distribution map generated by two aerial surveys of the same green-tide patch. (For interpretation of the references to color in this figure legend, the reader is referred to the Web version of this article.)

For the same green algae patch (Fig. 6a), UAV aerial photography missions were performed twice. The first aerial photography period was 13: 04: 55-13: 07: 38 on June 19, 2019 (Beijing time); the second was 13:08:26-13:11:42 of the same day. After being extracted by the RGBFAI, the distribution map of the green algae patch for both photography periods was generated (Fig. 6 b). Unlike stationary subjects, the position of the floating green algae constantly changed during the aerial photography period, thus, the shape of the green algae patches in the orthographic image may differ slightly from the true shape. To reduce this error and measure the displacement more accurately, this study selected six typical sub-patches of the green algae patches in sequence from north to south. The centroids of the six sub-patches were generated (Fig. 6b, A-G).

According to the capture time recorded in the aerial photographs, where the six centroids were located, the drift time (t) of the six centroids was calculated. The displacement (S) and direction (Dir) of six centroids are measured using the Arcmap software, and the drift velocity of six centroids can be calculated. Taking their average value as the drift speed $\left(\mathrm{V}_{\mathrm{ave}}\right)$ and direction $\left(\mathrm{Dir}_{\text {ave }}\right)$ of the entire green algae patch, the calculation formulas are as follows:

$\mathrm{V}_{\text {ave }}=\frac{1}{6} *\left(\sum_{\mathrm{i}=\mathrm{A}}^{\mathrm{F}} \frac{\mathrm{S}_{\mathrm{i}}}{\mathrm{t}_{\mathrm{i}}}\right)$

$\operatorname{Dir}_{\text {ave }}=\arctan \left(\frac{\sum_{\mathrm{i}=\mathrm{A}}^{\mathrm{F}} \mathrm{V}_{\mathrm{i}} * \sin \operatorname{Dir}_{\mathrm{i}}}{\sum_{\mathrm{i}=\mathrm{A}}^{\mathrm{F}} \mathrm{V}_{\mathrm{i}} * \cos D i r_{\mathrm{i}}}\right)$

where $i$ represent the sub-patches A-F. S, t, and Dir represent drift displacement, time, and direction, respectively, and their results are shown in the table inserted in Fig. $6 \mathrm{~b}$. Finally, the drift speed and direction of the entire green tide patch are approximate $0.26 \mathrm{~m} / \mathrm{s}$ and northeast $\left(17.1^{\circ}\right.$ from North). The calculated $\mathrm{Dir}_{\text {ave }}$ value is in line with the actual drift direction of the Yellow Sea green-tide reported in some previous studies. 


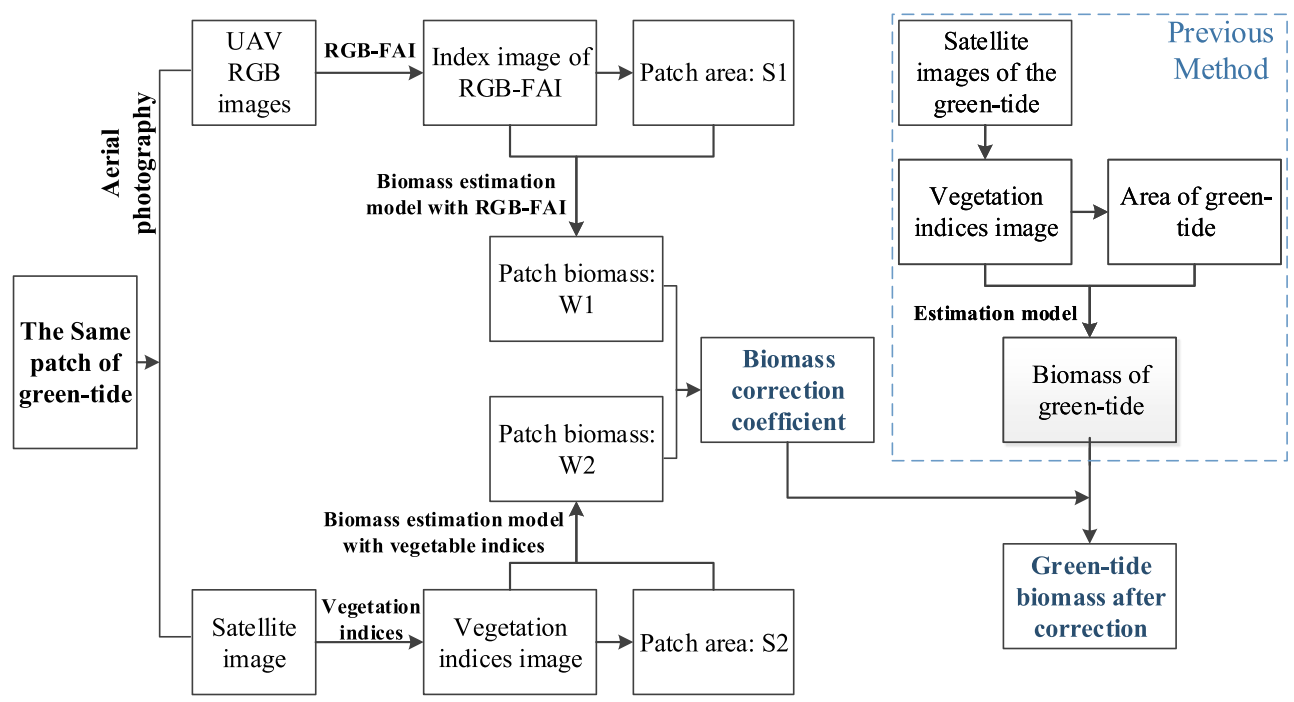

Fig. 7. Flowchart of an improved estimation method using the UAV remote sensing and RGB-FAI to correct the results of previous method.

\subsection{Implications for biomass estimation}

Two biomass estimation methods of green algae attached to the Porphyra yezoensis raft on the Subei Shoal have been proposed as the source of the Yellow Sea green-tide, based on UAV remote sensing etc. (Jiang et al., 2020). The biomass of floating green algae in the sea has been a critical parameter for green-tide research, salvaging, and prevention; however, its monitoring lacks accurate measurements (Wang et al., 2018). Previous researches have used ground experiments to establish the relationship between biomass per unit area and vegetation indices. Based on this relationship, a biomass estimation model for floating green-tide was constructed and applied to satellite remote sensing images to obtain biomass estimations (Hu et al., 2017). The estimation model was based on field spectral data. There were many differences between the satellite remote sensing data and field measurements, including atmospheric correction and mixed pixels (Xiao et al., 2017), which have affected the biomass estimation accuracy. The spectrum of UAV images is almost unaffected by the atmosphere and approximates the ground measurements (Flynn and Chapra, 2014). Therefore, UAV remote sensing can act as a bridge between field measurement and satellite remote sensing. Using UAV and satellite remote sensing to extract the same patch of green algae, a correction coefficient for satellite remote sensing estimation result is calculated with the biomass estimation model. Then, using the correction coefficient, a higher-precision biomass result of the green-tide is achieved (Fig. 7).

\section{Conclusion}

Due to the lack of NIR and SWIR bands, the design of the FAI and VBFAH for detecting green algal blooms could not be extended to the UAVbased RGB sensor despite its advantages over satellite sensors in terms of use-flexibility and spatial resolution. A novel index was introduced to detect green-tide in the Yellow Sea based on RGB images taking by shipborne UAV. RGB-FAI is defined as the difference between the reflectance in the green band and a baseline formed by the blue and red bands. The results showed that RGB-FAI excelled in monitoring floating green algae, especially in the decline phase even under a hazy atmosphere. The application of the new RGB-FAI to bi-temporal UAV-based orthophoto map successfully revealed green-tide drift velocity. These findings are also useful in estimating green-tide biomass. Due to the centimeter spatial resolution, high-flexibility, and little-to-no atmospheric interference of the UAV data, the RGB-FAI could be reliably used in highprecision monitoring of green-tide in the Yellow Sea. The findings can expand the applications of UAV remote sensing and provide a new method for marine environmental research.

\section{CRediT authorship contribution statement}

Xiaopeng Jiang: Conceptualization, Methodology, Data curation, Visualization, Investigation, Writing - original draft. Meng Gao: Writing - review \& editing, Funding acquisition. Zhiqiang Gao: Conceptualization, Resources, Validation, Supervision, Funding acquisition.

\section{Declaration of competing interest}

The authors declare that they have no known competing financial interests or personal relationships that could have appeared to influence the work reported in this paper.

The authors declare the following financial interests/personal relationships which may be considered as potential competing interests:

\section{Acknowledgement}

This work was supported from NSFC fund project (41876107), the NSFC-Shandong joint fund project (U1706219) and the National Key R\&D Program of China (2019YFD0900705). MG was funded by the Key Deployment Project of Center for Ocean Mega-Science, Chinese Academy of Sciences (COMS2019J02) and the Key Research Program of Frontier Science, Chinese Academy of Sciences (ZDBS-LY-7010).

\section{Appendix A. Supplementary data}

Supplementary data to this article can be found online at https://doi. org/10.1016/j.ecss.2020.106943.

\section{References}

Bao, M., Guan, W., Yang, Y., Cao, Z., Chen, Q., 2015. Drifting trajectories of green algae in the western Yellow Sea during the spring and summer of 2012. Estuarine. Coastal and Shelf Science 163, 9-16.

Bellvert, J., Zarco-Tejada, P.J., Girona, J., Fereres, E., 2014. Mapping crop water stress index in a 'Pinot-noir' vineyard: comparing ground measurements with thermal remote sensing imagery from an unmanned aerial vehicle. Precis. Agric. 15, 361-376.

Bendig, J., Yu, K., Aasen, H., Bolten, A., Bennertz, S., Broscheit, J., Gnyp, M.L., Bareth, G., 2015. Combining UAV-based plant height from crop surface models, visible, and near infrared vegetation indices for biomass monitoring in barley. Int. J. Appl. Earth Obs. Geoinf. 39, 79-87.

Chen, X., Zhang, J., Cui, T., Song, P., 2018. Extraction of the green tide drift velocity in the Yellow Sea based on GF-4. Acta Oceanol. Sin. 40, 29-38. 
Cui, T., Zhang, J., Zhi, L., Jia, Y.-J., Zhao, W., Wang, Z.-L., Meng, J., 2012. Satellite monitoring of massive green macroalgae bloom (GMB): imaging ability comparison of multi-source data and drifting velocity estimation. International Journal of Remote Sensing - INT J REMOTE SENS 33, 5513-5527.

Erdelj, M., Król, M., Natalizio, E., 2017. Wireless Sensor Networks and Multi-UAV systems for natural disaster management. Comput. Network. 124, 72-86.

Fairman, H.S., Brill, M.H., Hemmendinger, H., 1997. How the CIE 1931 color-matching functions were derived from Wright-Guild data. Color Res. Appl. 22, 11-23.

Fallati, L., Polidori, A., Salvatore, C., Saponari, L., Savini, A., Galli, P., 2019. Anthropogenic Marine Debris assessment with Unmanned Aerial Vehicle imagery and deep learning: a case study along the beaches of the Republic of Maldives. Sci. Total Environ. 693, 133581.

Flynn, K.F., Chapra, S.C., 2014. Remote sensing of submerged aquatic vegetation in a shallow non-turbid river using an unmanned aerial vehicle. Rem. Sens. 6, 12815-12836.

Hassan, M.A., Yang, M., Fu, L., Rasheed, A., Zheng, B., Xia, X., Xiao, Y., He, Z., 2019. Accuracy assessment of plant height using an unmanned aerial vehicle for quantitative genomic analysis in bread wheat. Plant Methods 15, 37.

Hu, C., 2009. A novel ocean color index to detect floating algae in the global oceans. Rem. Sens. Environ. 113, 2118-2129.

Hu, L., Hu, C., Ming-Xia, H.E., 2017. Remote estimation of biomass of Ulva prolifera macroalgae in the Yellow Sea. Rem. Sens. Environ. 192, 217-227.

Huete, A., Justice, C., Van Leeuwen, W., 1999. MODIS vegetation index (MOD13). Algorithm theoretical basis document 3.

Hunt, E.R., Cavigelli, M., Daughtry, C.S.T., McMurtrey, J.E., Walthall, C.L., 2005. Evaluation of digital photography from model aircraft for remote sensing of crop biomass and nitrogen status. Precis. Agric. 6, 359-378.

Jiang, X., Gao, Z., Zhang, Q., Wang, Y., Tian, X., Shang, W., Xu, F., 2020. Remote sensing methods for biomass estimation of green algae attached to nursery-nets and raft rope. Mar. Pollut. Bull. 150, 110678.

Kim, D.-W., Min, T.-S., Kim, Y., Silva, R.R., Hyun, H.-N., Kim, J.-S., Kim, K.-H., Kim, H.J., Chung, Y.S., 2019. Sustainable agriculture by increasing nitrogen fertilizer efficiency using low-resolution camera mounted on unmanned aerial vehicles. Int. J. Environ. Res. Publ. Health 16, 3893.

Liu, D., Keesing, J.K., Dong, Z., Zhen, Y., Di, B., Shi, Y., Fearns, P., Shi, P., 2010. Recurrence of the world's largest green-tide in 2009 in Yellow Sea, China: Porphyra yezoensis aquaculture rafts confirmed as nursery for macroalgal blooms. Mar. Pollut. Bull. 60, 1423-1432.

Liu, D., Keesing, J.K., He, P., Wang, Z., Shi, Y., Wang, Y., 2013. The world's largest macroalgal bloom in the Yellow Sea, China: formation and implications. Estuar. Coast Shelf Sci. 129, 2-10.

Liu, D., Keesing, J.K., Xing, Q., Shi, P., 2009. World's largest macroalgal bloom caused by expansion of seaweed aquaculture in China. Mar. Pollut. Bull. 58, 888-895.

Liu, F., Pang, S.J., Zhao, X.B., Hu, C.M., 2012. Quantitative, molecular and growth analyses of Ulva microscopic propagules in the coastal sediment of Jiangsu province where green tides initially occurred. Mar. Environ. Res. 74, 56-63.

Liu, X.-q., Wang, Z.-1., Xin, M., Li, Y., He, X.-p., 2016a. Study on process of nutrient release during the decay of Ulva Prolifera. Mar. Environ. Sci. 35, 801-805+813.

Liu, X., Wang, Z., Zhang, X., 2016b. A review of the green tides in the Yellow Sea, China. Mar. Environ. Res. 119, 189-196.

Luo, Y., Jian-Hua, X.U., Yue, W.Z., 2005. Research on vegetation indices based on the remote sensing images. Ecologic Science 24, 75-79.

Meyer, G.E., Neto, J.C., 2008. Verification of color vegetation indices for automated crop imaging applications. Comput. Electron. Agric. 63, 282-293.

Oyama, Y., Fukushima, T., Matsushita, B., Matsuzaki, H., Kamiya, K., Kobinata, H., 2015 Monitoring levels of cyanobacterial blooms using the visual cyanobacteria index (VCI) and floating algae index (FAI). Int. J. Appl. Earth Obs. Geoinf. 38, 335-348.
Pontius, R.G., Millones, M., 2011. Death to Kappa: birth of quantity disagreement and allocation disagreement for accuracy assessment. Int. J. Rem. Sens. 32, 4407-4429.

Rosenfield, G.H., Fitzpatrick-Lins, K., 1986. A coefficient of agreement as a measure of thematic classification accuracy. Photogramm. Eng. Rem. Sens. 52, 223-227.

Rouse, J., Haas, R., Schell, J., Deering, D., 1974. Monitoring Vegetation Systems in the Great Plains with ERTS, vol. 1. NASA Special Publication.

Smetacek, V., Zingone, A., 2013. Green and golden seaweed tides on the rise. Nature 504, 84.

Son, Y.B., Min, J.-E., Ryu, J.-H., 2012. Detecting massive green algae (Ulva prolifera) blooms in the Yellow Sea and east China sea using geostationary ocean color imager (GOCI) data. Ocean Sci. J. 47, 359-375.

Tian, H., Liu, Q., I.Goes, J., Gomes, H.d.R., Yang, M., 2020. Temporal and spatial changes in chlorophyll-a concentrations in the Yellow Sea from 2002 to 2008 based on Modis data. Mar. Sci. Bull. 39, 101-110.

Wang, X., Wang, M., Wang, S., Wu, Y., 2015a. Extraction of vegetation information from visible unmanned aerial vehicle images. Trans. Chin. Soc. Agric. Eng. 31, 152-157+ $159+158$.

Wang, Z., Jie, X., Fan, S., Yan, L., Liu, X., Liu, D., 2015b. Who made the world's largest green tide in China?-an integrated study on the initiation and early development of the green tide in Yellow Sea: green tide in Yellow Sea of China. Limnol. Oceanogr. 60, 1105-1117.

Wang, Z.L., Fu, M.Z., Xiao, J., Zhang, X.L., Song, W., 2018. Progress on the study of the Yellow Sea green tides caused by Ulva prolifera. Acta Oceanol. Sin. 40, 1-13.

Xiao, Y.F., Zhang, J., Cui, T.W., Gong, J.L., Xia, S.Z., Liu, R.J., Qing, P., Mu, B., 2017. Spectral characteristics and estimation models of floating green tide biomass on sea surface. Acta Opt. Sin. 37, 346-354.

Xing, C., Wang, J., Xu, Y., 2010. A method for building a mosaic with UAV images. Int. J. Inf. Eng. Electron. Bus. 1, 9-15.

Xing, Q., Hu, C., 2016. Mapping macroalgal blooms in the Yellow Sea and East China Sea using HJ-1 and Landsat data: application of a virtual baseline reflectance height technique. Rem. Sens. Environ. 178, 113-126.

Yan, T., Yu, R.C., Zhou, M.J., Sun, S., 2018. Mechanism of massive formation and prevention strategy against large-scale green tides in the south Yellow Sea. Oceanol. Limnol. Sinica 49, 950-958.

Yu, R.C., Sun, S., Yan, T., Zhou, M.J., 2018. Progresses and perspectives on green-tide studies in the yellow sea. Oceanol. Limnol. Sinica 49, 942-949.

Zhang, Q.-C., Yu, R.-C., Chen, Z.-F., Qiu, L.-M., Wang, Y.-F., Kong, F.-Z., Geng, H.-X., Zhao, Y., Jiang, P., Yan, T., Zhou, M.-J., 2018. Genetic evidence in tracking the origin of Ulva prolifera blooms in the Yellow Sea, China. Harmful Algae 78, 86-94.

Zhang, X., Zhang, F., Qi, Y., Deng, L., Wang, X., Yang, S., 2019. New research methods for vegetation information extraction based on visible light remote sensing images from an unmanned aerial vehicle (UAV). Int. J. Appl. Earth Obs. Geoinf. 78, 215-226.

Zhao, J., Jiang, P., Liu, Z.Y., Wei, W., Lin, H.Z., Fuchao, L.I., Wang, J.F., Qin, S., 2013. The Yellow Sea green tides were dominated by one species, Ulva (Enteromorpha) prolifera, from 2007 to 2011. Chin. Sci. Bull. 58, 2298-2302.

Zhao, J., Jiang, P., Qiu, R., Ma, Y., Wu, C., Fu, H., Chen, H., Li, F., 2018. The Yellow Sea green tide: a risk of macroalgae invasion. Harmful Algae 77, 11-17.

Zheng, X., Gao, Z., Xu, F., Ning, J., Song, D., Zheng, B., 2017. Study of remote sensing monitoring and comparisong of green tide in the Yellow Sea based on VB-FAH index. Mar. Sci. 41, 71-79.

Zhong, S., Ding, Y., Li, Z., Liu, A., Tong, Z., Wang, Q., 2013. Error analysis on enteromorpha prolifera monitoring using MODIS data. Remote Sens. Inf. 28, 40-44.

Zhou, M.-J., Liu, D.-Y., Anderson, D.M., Valiela, I., 2015. Introduction to the special issue on green tides in the Yellow Sea. Estuar. Coast Shelf Sci. 163, 3-8. 